\title{
SUAVIZAÇÃO DA FRONTEIRA DEA DE EFICIÊNCIA: ESTADO DA ARTE E PERSPECTIVAS FUTURAS
}

\author{
Juliana de Castro Reis \\ Universidade Federal Fluminense \\ Rua Passos da Pátria, 156 - Niterói - RJ \\ juliana.dcreis@gmail.com \\ Luana Brandão \\ Universidade Federal Fluminense \\ Rua Passos da Pátria, 156 - Niterói - RJ \\ luanabrandao@id.uff.br \\ João Carlos Correia Baptista Soares de Mello \\ Universidade Federal Fluminense \\ Rua Passos da Pátria, 156 - Niterói - RJ \\ jccbsmello@id.uff.br
}

\begin{abstract}
RESUMO
Devido às propriedades da fronteira DEA de eficiência, que é linear por partes, o modelo dos multiplicadores admite múltiplos pesos ótimos e, por consequência, múltiplas soluções ótimas para as DMUs extremo-eficientes (vértices das faces da fronteira), o que impossibilita o conhecimento das razões de substituição (tradeoffs) e a aplicação de algumas técnicas, como a Avaliação Cruzada. Uma solução para esse problema é a substituição da fronteira original por uma fronteira suavizada, que seja próxima da fronteira original e que tenha derivadas contínuas em todos os pontos. Apesar de ter sido desenvolvida há algum tempo, não há na literatura muitos trabalhos que discutam as propriedades ou mesmo a aplicação da técnica de Suavização da Fronteira. Assim, o objetivo deste trabalho é realizar uma revisão sistemática de estudos relacionados à Suavização da Fronteira DEA, examinando as melhorias metodológicas propostas, os modelos desenvolvidos, as aplicações numéricas e as lacunas ainda existentes.
\end{abstract}

Palavra-chave: Fronteira de eficiência, Suavização da fronteira, Fronteira suavizada, Múltiplos pesos ótimos, Análise Envoltória de Dados.

\begin{abstract}
Due to the DEA efficiency frontier properties, which is of piecewise linear, the multiplier model allows multiple optimal weights and, as a result, multiple optimal solutions for extremely efficient DMUs (vertices of the frontier). This fact makes it impossible to understand the tradeoffs and to apply some techniques, such as cross-evaluation. One solution to this problem is to replace the original frontier with a smoothed frontier, close to the original frontier and with continuous derivations at all points. Although it has been developed for some time, there are not many studies in the literature that discuss the properties or even application of the Frontier Smoothing technique. Thus, the aim of this
\end{abstract}


paper is to conduct a systematic review of studies related to DEA Frontier Smoothing, examining proposed methodological improvements, developed models, numerical applications and remaining gaps.

Keywords: Efficiency frontier, Frontier Smoothing, Smoothed frontier, Multiple optimal Weights, Data Envelopment Analysis.

\section{Como Citar:}

REIS, Juliana de Castro; BRANDÃO, Luana; SOARES DE MELLO, João Carlos Correia Baptista. Suavização da fronteira DEA de eficiência: estado da arte e perspectivas futuras. In: SIMPÓSIO DE PESQUISA OPERACIONAL E LOGÍSTICA DA MARINHA, 19., 2019, Rio de Janeiro, RJ. Anais [...]. Rio de Janeiro: Centro de Análises de Sistemas Navais, 2019.

\section{INTRODUÇÃO}

Já é difundido na literatura que a formulação dos Multiplicadores dos modelos DEA implica na obtenção de múltiplos pesos ótimos, e como consequência, múltiplas soluções ótimas, para as DMUs extremo-eficientes. Isso porque, devido às características da fronteira de eficiência que é linear por partes, as DMUs localizadas nos vértices da fronteira não possuem derivadas únicas.

Essa multiplicidade de soluções ótimas é frequentemente ignorada nas avaliações de eficiência ou, às vezes, uma breve referência é feita. Na grande maioria dos casos são utilizados os primeiros valores encontrados pelo PPL para os pesos das variáveis de cada DMU (THANASSOULIS, 1993).

Porém, o problema de não se obter um conjunto único de pesos impacta, ou até mesmo inviabiliza, a aplicação de algumas técnicas, como a Avaliação Cruzada, ou a utilização de DEA em problemas multicritério. Além disso, não é possível determinar as razões de substituição (tradeoffs) ou a importância que cada DMU atribui à determinada variável no cálculo de sua eficiência.

Para transpor esse empecilho, Charnes et al. (1985) propuseram a utilização arbitrária de um valor único para as derivadas através do cálculo de uma média ponderada, com base nos baricentros das hipersuperfícies concorrentes. Em outra vertente, o modelo da superficiência desenvolvido por Andersen \& Petersen (1993) também fornece um conjunto único de pesos para cada DMU. No entanto, esse modelo tem várias desvantagens, como o índice de eficiência não estar contido no intervalo entre 0 e 1 e a possibilidade de levar a um PPL inviável para algumas DMUs extremo-eficientes.

Em seu modelo de Avaliação Cruzada, Doyle \& Green (1995) também abordaram o problema de escolher uma solução a partir de uma infinidade de soluções ótimas. Como solução, propuseram a utilização de modelos com funções objetivo adicionais, que chamaram de modelo agressivo e modelo benevolente.

Por fim, Soares de Mello, Lins \& Gomes (2002) propuseram uma metodologia de Suavização da Fronteira DEA, que consiste em substituir a fronteira DEA original por outra, com derivadas contínuas em todos os pontos. Apesar de ter sido desenvolvida há algum tempo, não há na literatura muitos trabalhos que discutam as propriedades ou mesmo a aplicação dessa técnica. Os poucos estudos publicados contribuíram para a evolução da metodologia, implementando melhorias no modelo de Suavização da Fronteira e expandindo a metodologia para os casos de múltiplos inputs e múltiplos outputs.

O objetivo deste trabalho é realizar uma revisão sistemática de estudos relacionados à Suavização da Fronteira DEA, examinando as melhorias metodológicas propostas, os 
modelos desenvolvidos, as aplicações numéricas e as lacunas ainda existentes. Este trabalho também visa destacar algumas direções para possíveis estudos futuros e inspirar pesquisadores a explorar novas áreas de aplicação e a resolução de problemas práticos da metodologia.

Na próxima seção é feita uma explanação sobre a metodologia de Suavização da Fronteira, apresentando o conceito e sua formulação geral. Em seguida, é realizada uma revisão da literatura dos modelos desenvolvidos para retornos variáveis de escala (BCC) e para retornos constantes de escala (CCR). Por fim, a Seção 3 descreve as considerações finais do artigo e algumas propostas para trabalhos futuros.

\section{SUAVIZAÇÃO DA FRONTEIRA DE EFICIÊNCIA}

\subsection{CONCEITO, FORMULAÇÃO GERAL E APLICAÇÕES}

Na Suavização da Fronteira de eficiência o que se busca é um método de resolução da ambigüidade na determinação dos pesos, de tal forma que cada DMU tenha um único conjunto de pesos, permitindo conhecer a importância relativa de cada entrada ou saída (SOARES DE MELLO; LINS; GOMES, 2002).

Para resolver este problema, é necessário interpretar os pesos no modelo dual, isto é, no Modelo do Envelope. Conforme destacado por Lins \& Angulo Meza (2000), através do Teorema das Folgas Complementares é possível mostrar que os pesos correspondem aos coeficientes do hiperplano que é tangente a cada ponto da fronteira de eficiência, chamado de hiperplano de suporte.

Então, o problema de determinar um único conjunto de pesos no Modelo dos Multiplicadores é equivalente a determinar um hiperplano tangente no Modelo do Envelope, para o qual é necessário calcular as derivadas parciais em todos os pontos da fronteira. Como os únicos pontos na fronteira onde não há inclinação são as DMUs extremo-eficientes, que representam "cantos" da hipersuperfície, o que se busca é suavizar esses cantos (SOARES DE MELLO; LINS; GOMES, 2002).

Soares de Mello, Lins \& Gomes (2002) propuseram substituir a fronteira calculada no modelo DEA clássico (linear por partes) por outra que aceite um hiperplano tangente em todos os seus pontos, mas que mantenha a maioria das propriedades originais da fronteira DEA, por exemplo, que a nova fronteira e a fronteira original sejam determinadas pelas mesmas DMUs eficientes.

Para viabilizar tal solução, não apenas a função que representa a nova fronteira deve estar próxima da fronteira original (para que as eficiências calculadas não sejam substancialmente diferentes), mas suas derivadas também devem ter valores semelhantes aos originais, sempre que existirem. Dessa forma, Soares de Mello, Lins \& Gomes (2002) precisaram determinar uma métrica que considerasse simultaneamente a distância entre duas funções e suas derivadas, com o intuito de determinar a proximidade das duas fronteiras.

Em um modelo bidimensional, uma região da fronteira contendo duas DMUs eficientes consecutivas é um segmento de linha reta. Como o segmento de linha reta é o menor comprimento de arco entre dois pontos, qualquer outra curva conectando dois pontos da fronteira terá um comprimento de arco maior, que será tanto maior quanto maior forem as divergências em relação à linha reta. Além disso, quanto mais oscilante for a função na vizinhança do segmento de reta (implicando a existência de derivadas cujo valor é substancialmente diferente), maior o comprimento do arco.

Portanto, ao utilizar as diferenças entre comprimentos de arco para determinar se a fronteira suavizada está localizada na vizinhança da fronteira original, é possível levar em consideração os valores da função e de suas derivadas. Como a fronteira original é composta 
por um conjunto de segmentos de retas conectados, e como o segmento de reta é a menor distância euclidiana entre dois pontos, basta minimizar o comprimento do arco da fronteira suavizada para garantir sua proximidade com a fronteira original (SOARES DE MELLO; LINS; GOMES, 2002).

Vale destacar que a diferença do comprimento de arco não gera uma métrica. Isso porque, em alguns casos específicos, é possível haver funções distintas onde a diferença entre os seus comprimentos de arco é zero. Por esse motivo, não se deve falar em distância entre as duas fronteiras, mas apenas de proximidade ou de pertencer a uma vizinhança.

O problema de suavizar a fronteira DEA, então, consiste em procurar uma função que minimize o comprimento do arco (ou sua generalização n-dimensional), que contém as DMUs pareto eficientes e que possui derivadas parciais de segunda ordem em todos os seus pontos. Para facilitar sua resolução, o quadrado do comprimento do arco pode ser minimizado sem alterar os resultados.

Seguindo esse princípio, Soares de Mello, Lins \& Gomes (2002) propuseram a formulação geral da Suavização da Fronteira apresentada em (I), onde xi representa os inputs e $F(X)$ é o output. Este é um problema do Cálculo Variacional (minimização de uma função), que pode ser reduzido à solução de uma equação diferencial parcial, dentro de determinadas condições de limite.

$\min L=\int_{R}\left[1+\sum_{i}\left(\frac{\partial F}{\partial x_{i}}\right)^{2}\right] d S$

sa

$F\left(\vec{X}_{j}\right)=\operatorname{output}\left(\vec{X}_{j}\right), \forall \vec{X}_{j} \in E=\{\vec{X}: \vec{X}$ é uma DMU pareto eficiente $\}$

$\forall \vec{X}_{j}, \exists \frac{\partial F}{\partial x_{i}}$

Em seu estudo, Soares de Mello, Lins \& Gomes (2002) concluíram ainda que o problema de determinar uma fronteira suavizada que mais se aproxima da fronteira original, utilizando uma topologia baseada no comprimento do arco, é impossível. O que significa dizer que, dada uma fronteira suavizada, sempre haverá a possibilidade de encontrar uma outra fronteira (e, portanto, uma infinidade delas) que será uma aproximação ainda melhor da original. Porém, o fato de não ser possível encontrar a melhor aproximação não significa que não existem boas aproximações da fronteira original que possam ser utilizadas.

\subsection{MODELOS COM RETORNOS VARIÁVEIS DE ESCALA (BCC)}

Soares de Mello, Lins \& Gomes (2002) iniciaram o estudo da Suavização da Fronteira de eficiência para os casos de retornos variáveis de escala, analisando o modelo BCC com um input e um output, onde as faces da fronteira são segmentos de reta. Nos modelos BCC (BANKER; CHARNES; COOPER, 1984), com retornos variáveis de escala, a fronteira de eficiência é convexa e requer que $\frac{\partial^{2} F}{\partial x_{i}^{2}} \leq 0$.

Para determinar uma boa aproximação, deve-se minimizar uma função escolhida dentro de uma determinada família, chamada de funções aproximantes. Segundo Soares de Mello, Lins \& Gomes (2002), a escolha da função aproximante determina a complexidade dos cálculos e a qualidade da solução. Dessa maneira, os autores escolheram trabalhar com funções aproximantes quadráticas. O principal motivo dessa escolha foi o fato de que as 
parábolas possuem a segunda derivada com sinal constante, o que significa que são sempre côncavas ou sempre convexas, facilitando a aplicação de restrição de convexidade da fronteira.

Ao trabalhar com funções aproximantes quadráticas, se houver $n$ DMUs pareto eficientes, haverá $n-1$ segmentos de reta unindo essas DMUs e, portanto, $n-1$ funções da forma $y i=a_{i} x^{2}+b_{i} x+c_{i}$. Como cada função possui três parâmetros, serão $3(n-1)$ variáveis de decisão introduzidas no problema de minimização. Também devem ser consideradas no modelo as restrições de existência de derivadas, necessárias apenas nas DMUs intermediárias, onde as fronteiras à esquerda e à direita são definidas por funções distintas. Logo, devem existir $n$-2 restrições de suavização.

O modelo de Suavização da Fronteira proposto por Soares de Mello, Lins \& Gomes (2002) para o caso de um input e um output com retornos variáveis de escala é apresentado em (II).

$\min \sum_{i=1}^{p-1} \int_{x_{i}}^{x_{i+1}}\left\{1+\left[\left(a_{i} x^{2}+b_{i} x+c_{i}\right)\right]^{2}\right\} d x$

st

$a_{1} x_{1}^{2}+b_{1} x_{1}+c_{1}=y_{1}\left(\right.$ para a $D M U_{1} i$

$a_{p-1} x_{p}^{2}+b_{p-1} x_{p}+c_{p-1}=y_{p}\left(\right.$ para a $D M U_{p} i$

$a_{i-1} x_{i}^{2}+b_{i-1} x_{i}+c_{i-1}=y_{i}=a_{i} x_{i}^{2}+b_{i} x_{i}+c_{i}\left(\right.$ para a $D M U_{i}, \forall i(2, \ldots, p-1) i$

$2 a_{i-1} x_{i}+b_{i-1}=2 a_{i} x_{i}+b_{i}$ (fronteira suavizada para a $D M U_{i}, \forall i(2, \ldots, p-1) \dot{i}$

$a_{i} \leq 0$ (convexidade da fronteira)

Neste modelo, as duas primeiras restrições garantem que a nova fronteira passe pelas DMUs com menor input e maior output. As próximas restrições garantem a continuidade da fronteira e de suas derivadas nas DMUs extremo-eficientes, e a última restrição garante a convexidade da fronteira.

Uma das lacunas deixadas por esse modelo é a possibilidade de ocorrer a inviabilidade do problema de suavização nos casos que houver mais de uma DMU não extremo-eficiente. Isso porque a existência de três pontos colineares anula o coeficiente do termo quadrático da parábola.

Mais tarde, Soares de Mello et al. (2004) desenvolveram a formulação da Suavização da Fronteira para modelos tridimensionais, com dois inputs e um output. A principal mudança proposta em relação ao trabalho percursor foi a utilização de uma função aproximante polinimial única para toda a fronteira (ao invés de utilizar uma função aproximante polinomial de $2^{\circ}$ grau para cada região da fronteira).

Dessa forma, para garantir que o número de restrições de igualdade seja inferior ao número de variáveis de decisão (coeficientes do polinômio), foi necessário trabalhar com funções polinomiais de grau mais elevado, como a apresentada em (III), onde $x$ e $y$ são os inputs e $Z$ representa o output.

$Z=a+b x+c y+d x^{2}+e x y+f y^{2}+g x^{3}+h x^{2} y+i x y^{2}+j y^{3}+k x^{4}+l x^{3} y+m x^{2} y^{2}+n x y^{3}+o y^{4}$

Outra mudança trazida pelo novo modelo é a garantia de que a fronteira suavizada 
contenha todas as DMUs extremo-eficientes, embora possa não conter DMUs eficientes que não sejam "cantos" da fronteira. A exclusão dessas DMUs visa para evitar as inviabilidades no problema de suavização apontado por Soares de Mello, Lins \& Gomes (2002).

Assim, a formulação proposta por Soares de Mello et al. (2004) para o modelo tridimensional é apresentada em (IV). A função objetivo é dada pela integral dupla, onde $y_{\min }, x_{\min }, y_{\max }$ e $x_{\max }$ representam o menor e o maior valor de cada input. A primeira restrição garante que as DMUs extremo-eficientes estejam contidas na fronteira suavizada. A segunda e a terceira restrições garantem a monotonicidade crescente da fronteira e, a última restrição garante a sua convexidade, impondo a condição de que todos os termos do polinômio devem ser convexos.

$\min \left\{\int_{x_{\min }}^{x_{\max }} \int_{y_{\min }}^{y_{\max }}\left[1+\left(\frac{\partial Z}{\partial x}\right)^{2}+\left(\frac{\partial Z}{\partial y}\right)^{2}\right] d y d x\right\}$

sa

$Z\left(x_{e f i}, y_{e f i}\right)=Z_{e f i}$

$\frac{\partial Z}{\partial x}\left(x_{\max }, y_{\max }\right) \geq 0$

$\frac{\partial Z}{\partial y}\left(x_{\max }, y_{\max }\right) \geq 0$

d, f, g, h, I, ... $\leq 0$

Soares de Mello et al. (2004) utilizaram este modelo em um estudo de caso sobre avaliação de eficiência de companhias aéreas brasileiras. Embora aplicado apenas para o caso de dois inputs e um output, o trabalho de Soares de Mello et al. (2004) poderia ser facilmente estendido para casos de maiores dimensões, desde que se tivesse um output e vários inputs, ou um input e vários outputs.

Partindo do mesmo raciocínio de utilizar um polinômio único para toda a fronteira, Nacif, Soares de Mello \& Angulo Meza (2009) desenvolveram uma formulação para tratar da multiplicidade simultânea de inputs e outputs em problemas de Suavização da Fronteira DEA de eficiência.

Para isso, Nacif, Soares de Mello \& Angulo Meza (2009) propuseram que a fronteira suavizada seja representada por uma equação que liga todos os inputs e outputs, onde os outputs são representados por um polinômio $P^{O}$ de grau $g_{O}$ e $m$ variáveis $z_{i}$, e os inputs são representados por um polinômio $P^{I}$ de grau $g_{I}$, a ser determinado, e $n$ variáveis $x_{\mathrm{i}}$.

Nesse modelo, a equação da fronteira suavizada será substituída por (V) e o os coeficientes dos polinômios serão determinados através do problema de programação não linear apresentado em (VI).

$P_{1, m}^{O}\left(z_{1} \ldots z_{m}\right)=P_{g, n}^{I}\left(x_{1} \ldots x_{m}\right)$ 
$\min \left\{\int_{x_{1 \min }}^{x_{1 \max }} \ldots \int_{x_{\text {min }}}^{x_{\max }}\left[1+\sum_{i=1}^{n} \sum_{j=1}^{m}\left(\frac{\partial Z_{j}}{\partial x_{i}}\right)^{2}\right] d x_{n} \ldots d x_{1}\right\}$

sa

$P_{1, m}^{O}\left(z_{1 e f i} \ldots z_{m e f i}\right)=P_{g, n}^{I}\left(x_{1 e f i} \ldots x_{m e f i}\right), \forall$ DMU extremo-eficiente

$\frac{\partial Z_{j}}{\partial x_{i}}\left(x_{1 \max }, x_{n \max }\right) \geq 0, \forall j=1 \ldots m, i=1 \ldots n, x_{i}$

$\frac{\partial^{2} Z_{j}}{\partial x_{i}^{2}} \leq 0, \forall j=1 \ldots m, i=1 \ldots n, x_{i}$

Em seu estudo, Nacif, Soares de Mello \& Angulo Meza (2009) determinaram ainda um algoritmo para auxiliar na determinação do grau do polinômio referente aos inputs e apresentaram um exemplo número para o caso de dois inputs e dois outputs.

Por fim, Brandão \& Soares de Mello (2017) identificaram deficiências nos modelos de suavização anteriores, que não garantiam completamente a convexidade da fronteira, propriedade essencial dos modelos BCC.

Além disso, também propuseram melhorias para que todas as projeções das DMUs, e consequentemente, seus valores de eficiência, sejam sempre não negativos. Segundo Brandão \& Soares de Mello (2017), apesar de estar presente nos modelos DEA clássicos, esta propriedade não foi assegurada nos modelos de suavização anteriores. Os autores também propuseram o uso do modelo de suavização melhorado para evitar distorções do BCC clássico, as chamadas DMUs eficientes por default, onde se uma DMU tiver o menor valor para qualquer input ou o maior valor para qualquer output, esta DMU é necessariamente considerada eficiente.

Para garantir a convexidade da fronteira, Brandão \& Soares de Mello (2017) eliminaram todos os termos cruzados da função polinomial que descreve a fronteira suavizada, ou seja, todos os termos cruzados foram considerados nulos. Assim, a função polinomial que descreve a fronteira para o caso de um output e múltiplos inputs, por exemplo, passa a ser conforme apresentado em (VII).

$Z=F(x, y)=a+b x+c y+d x^{2}+e y^{2}+f x^{3}+g y^{3}+\ldots$

Brandão \& Soares de Mello (2017) observaram que nos modelos com orientação a inputs pode acontecer o problema de alvos com valores negativos, quando os valores de inputs para uma DMU ineficiente são reduzidos até que alcance a fronteira. Para evitar as projeções com valores de inputs negativos, os autores impuseram a restrição que todos os inputs na fronteira suavizada devem ser maiores ou iguais a zero, para todos os valores de output.

Por fim, Brandão \& Soares de Mello (2017) trataram do problema das DMUs eficientes por default que, segundo os autores, afeta não só a avaliação dessas DMUs falsamente eficientes, como também impacta em todas as DMUs que estão localizadas ou que são projetadas em faces da fronteira que contém DMUs eficientes por default. Para solucionar esse problema as restrições de igualdade associadas as DMUs eficientes por default foram relaxadas. Assim, essas DMUs continuam fazendo parte do conjunto de possibilidades de produção do modelo de suavização, porém a nova fronteira de eficiência pode conter essas DMUs ou não. 
A nova formulação do modelo de Suavização da Fronteira DEA com retornos variáveis de escala proposta por Brandão \& Soares de Mello (2017) é apresentada em (VIII), para os casos com múltiplos inputs e um output. Neste modelo, a primeira restrição é referente ao tratamento das eficiências por default e a segunda restrição garante que a fronteira suavizada contenha todas as outras DMUs eficientes. O conjunto de restrições seguinte garante que os outputs são uma função crescente dos inputs e, por fim, a última restrição garante alvos positivos para todas as DMUs.

$\min \left\{\int_{x_{1 \min }}^{x_{1 \max }} \ldots \int_{x_{n \min }}^{x_{\max }} \sum_{i=1}^{n}\left(\frac{\partial F}{\partial x_{i}}\right)^{2} d x_{n} \ldots d x_{1}\right\}$

sa

$F\left(x_{1 e f i} \ldots, x_{n e f i}\right) \geq Z_{e f i}, \forall$ DMU eficiente por default

$F\left(x_{1 e f i} \ldots, x_{\text {nefi }}\right)=Z_{e f i}, \forall$ DMU eficiente

$\frac{\partial F}{\partial x_{i}}\left(x_{1 \text { max }} \ldots, x_{\text {nmax }}\right) \geq 0, \forall i=1, \ldots, n$

$c_{1}, \ldots, c_{n} d_{1}, \ldots, d_{n}, \ldots \leq 0 ; a \leq z_{\text {min }}$

Em (IX) é apresentado o modelo proposto para os casos com um input e múltiplos outputs.

$\min \left\{\int_{z_{1 \min }}^{z_{1 \max }} \ldots \int_{z_{\min }}^{z_{\max }} \sum_{i=1}^{m}\left(\frac{\partial H}{\partial z_{i}}\right)^{2} d z_{m} \ldots d z_{1}\right\}$

sa

$H\left(z_{1 e f i}, \ldots, z_{m e f i}\right) \leq X_{e f i}, \forall D M U$ eficiente por default

$H\left(z_{1 e f i} \ldots, z_{\text {mefi }}\right)=Z_{e f i}, \forall$ DMU eficiente

$\frac{\partial H}{\partial z_{j}}\left(z_{1 \text { min }} \ldots, z_{\text {mmin }}\right) \geq 0, \forall j=1, \ldots, m$

$c_{1}, \ldots, c_{m}, d_{1}, \ldots, d_{m}, \ldots \geq 0$

$H\left(z_{1 \text { min }} \ldots, z_{\text {mmin }}\right) \geq 0$

Assim como no trabalho de Soares de Mello et al. (2004), Brandão \& Soares de Mello (2017) aplicaram o novo modelo de suavização, após as melhorias propostas, em um estudo de caso para avalizar o desempenho operacional de companhias aéreas brasileiras.

Abaixo, a Figura 1 apresenta um esquema com a evolução da metodologia de Suavização da Fronteira DEA de eficiência para os modelos com retornos variáveis de escala ao longo dos anos. 


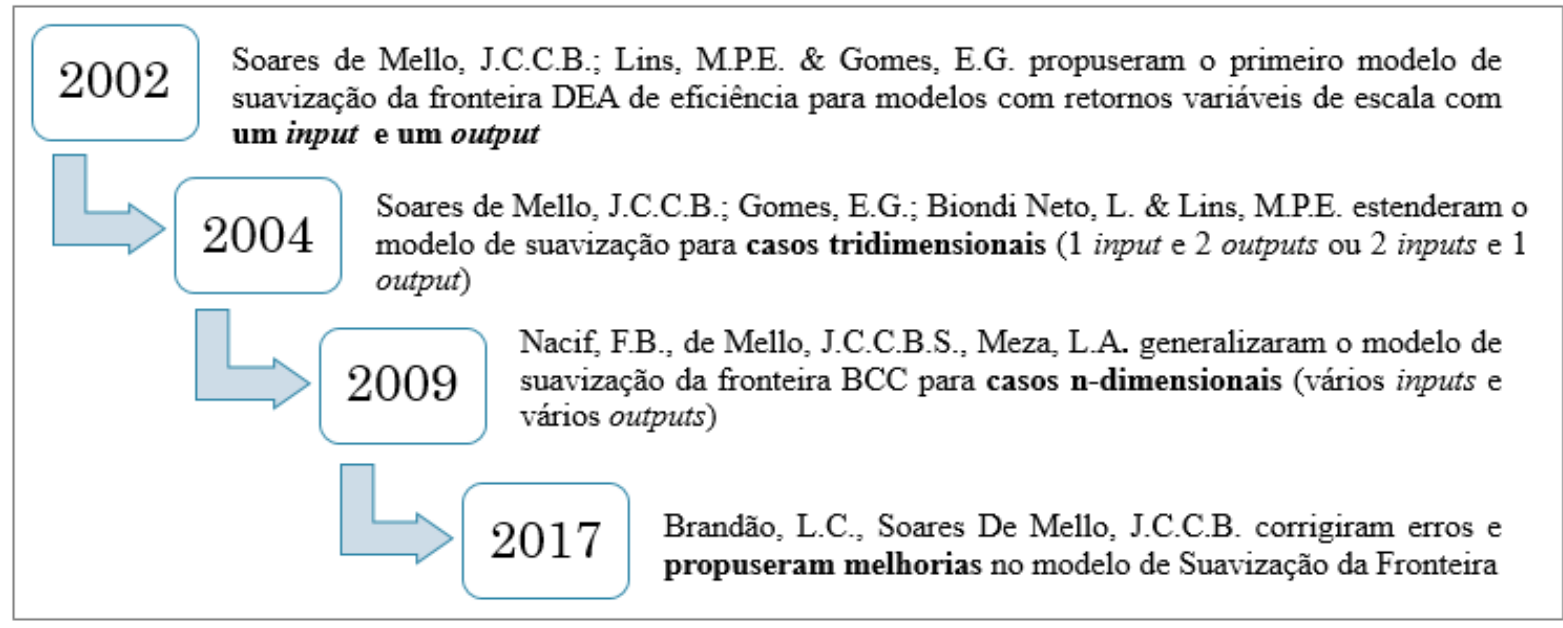

Figura 1 - Evolução dos modelos de suavização da fronteira DEA BCC

Em trabalho mais recente, Alvarenga Junior \& Lins (2018) aplicaram a metodologia de Suavização da Fronteira ao desenvolverem um modelo para seleção de materiais em um estoque gerenciado corporativamente, que inclui peças sobressalentes de alto valor. $\mathrm{O}$ objetivo do modelo é reduzir o valor emprenhado em estoques sem comprometer a confiabilidade das unidades operacionais das Refinarias brasileiras. A principal razão citada pelos autores para utilizar uma fronteira DEA suavizada é que a função da nova fronteira poderá ser utilizada para o cálculo direto da eficiência radial quando um novo material for adicionado ao estoque das refinarias, sem a necessidade de executar toda a programação matemática de DEA novamente.

\subsection{MODELOS COM RETORNOS CONSTANTES DE ESCALA (CCR)}

Para os modelos com retornos constantes de escala, a condição de convexidade é substituída pela condição de proporcionalidade, ou seja, aumentos nos inputs geram aumentos proporcionais nos outputs.

Isso significa dizer que $F(k \cdot X)=k \cdot F(X)$. Assim, a função $F$ que irá descrever a fronteira suavizada precisa ser homogênea e de primeiro grau. Esta propriedade dificulta sobremaneira a escolha de funções aproximantes para os modelos com retornos constantes de escala, mas por outro lado permite aproveitar as propriedades das funções homogêneas para simplificar o problema.

Em seu primeiro trabalho, Soares de Mello, Lins \& Gomes (2002) encontraram dificuldades devido à restrição imposta pelo Teorema de Euler. Dessa forma, naquele momento os autores não apresentaram uma proposta de fronteira suavizada para os modelos com retornos constantes de escala. Mesmo assim, destacaram que a função utilizada para descrever a fronteira suavizada deveria obedecer ao Teorema de Euler para funções homogêneas e uma restrição adicional deveria ser incluída no modelo CCR.

Com base na metodologia proposta mais tarde por Nacif, Soares de Mello \& Angulo Meza (2009) para modelos BCC, Pereira et al. (2015) propõem o uso de um polinômio único como aproximante da fronteira DEA CCR, definindo a função U descrita em (X) que relaciona os polinômios homogêneos de outputs e de inputs.

$U\left(x_{1}, X_{2}, \ldots, X_{n}, z_{1}, z_{2}, \ldots, z_{n}\right)=F g_{l, n}\left(X_{1}, X_{2}, \ldots, x_{n}\right)-H g_{o, m}\left(z_{1}, z_{2}, \ldots, z_{n}\right)$

onde, $F$ e $H$ representam os polinômios homogêneos de inputs e outputs, respectivamente; $g_{I}$ é o grau do polinômio dos inputs e $g$, o grau do polinômio dos outputs; $n$ 
representa o número de inputs e $m$ o número de outputs. Finalmente, $x_{i}$ e $z_{i}$ são os valores dos inputs e outputs das DMUs em estudo.

Como destacado pelos autores, o uso da função $U$ permite que as restrições de suavização sejam satisfeitas implicitamente pelas derivadas dessa função, que são facilmente calculadas. A seguir, a formulação (XI) representa o modelo DEA CCR suavizado proposto por Pereira et al. (2015), para quaisquer números de variáveis de entrada e de saída.

$\min \left\{\int_{x_{\min }}^{x_{1 \max }} \int_{x_{n \min }}^{x_{\max }} \ldots \int_{z_{\min }}^{z_{1 \max }} \int_{z_{\operatorname{mmin}}}^{z_{\operatorname{mmax}}}\left[1+\sum_{i=1}^{n}\left(\frac{\partial U}{\partial x_{i}}\right)^{2}+\sum_{i=1}^{m}\left(\frac{\partial u}{\partial z_{i}}\right)^{2}\right] d z_{m} \ldots d z_{1} d x_{m} \ldots d x_{1}\right\}$

sa

$U\left(x_{1 e f i} \ldots, x_{n e f i}, z_{1 e f i} \ldots, z_{m e f i}\right)=0, \forall$ DMU eficiente

$\frac{\partial U}{\partial x_{i}}\left(x_{1 \text { max }} \ldots, x_{n \text { max }}\right) \geq 0, \forall i=1, \ldots, n$

$\frac{\partial U}{\partial z_{i}}\left(z_{1 \min } \ldots, z_{n \min }\right) \leq 0, \forall i=1, \ldots, m$

$\frac{\partial U}{\partial x_{i}} \geq 0, \forall$ DMU extremo-eficiente

$\frac{\partial U}{\partial z_{i}} \leq 0, \forall$ DMU extremo-eficiente

A primeira restrição de (XI) garante que as DMUs extremo-eficientes estejam contidas na fronteira suavizada. A segunda restrição do modelo garante a monotonicidade crescente da fronteira para os inputs em seus pontos máximos, enquanto para os outputs é garantida através da terceira restrição, em seus pontos mínimos. Como salientado pelos autores, as duas últimas restrições são adicionadas para evitar a ocorrência de grandes variações na função aproximante definida em relação a fronteira original, garantindo a monotonicidade para inputs e outputs nas DMUs extremo-eficientes.

No entanto, o modelo desenvolvido por Pereira et al. (2015) garante o comportamento da nova fronteira apenas localmente, para as DMUs extremo-eficientes. Assim, o modelo desenvolvido para suavização da fronteira CCR é válido apenas dentro de um intervalo de calibração, definido pelos valores mínimos e máximos dos inputs e outputs de cada variável do estudo.

A principal lacuna deixada por proposta é que o comportamento da fronteira suavizada não é garantido em toda a sua extensão, podendo mudar a concavidade a partir de um determinado ponto. Por esse motivo, o modelo não garante o envelopamento de todas as DMUs do estudo, propriedade fundamental dos modelos DEA.

A Figura 2 apresenta um resumo dos desenvolvimentos da metodologia de Suavização da Fronteira DEA de eficiência para os modelos com retornos constantes de escala. Pode-se observar que, devido as dificuldades que a restrição de proporcionalidade impõe, há uma evolução menor da metodologia para os modelos DEA CCR, deixando importantes lacunas que precisam ser estudadas. 


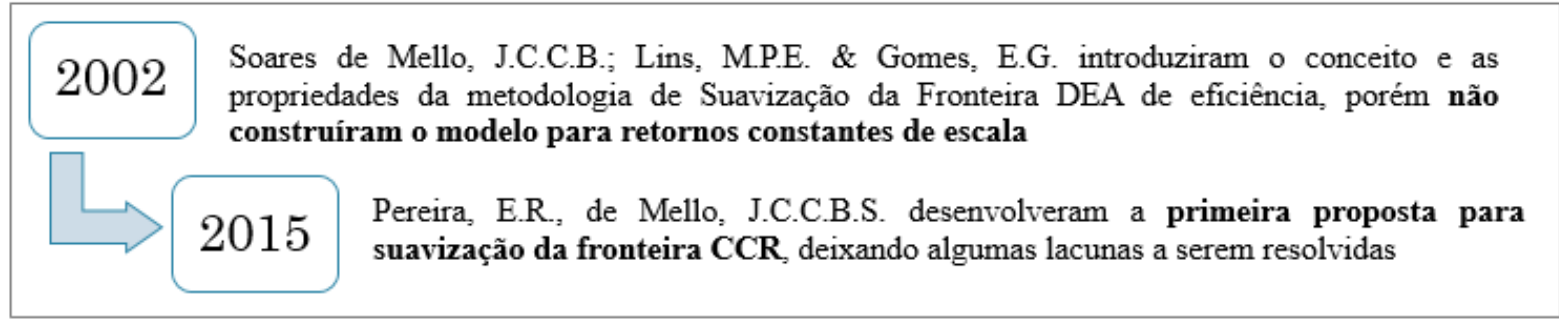

Figura 2 - Evolução dos modelos de suavização da fronteira DEA CC.

\section{CONSIDERAÇÕES FINAIS}

A metodologia de Suavização da Fronteira mostra-se eficiente para resolver o problema de múltiplos pesos ótimos das DMUs extremo-eficientes, apresentando vantagens em relação às propostas de outros autores para tratar do mesmo problema (essas vantagens foram listadas no trabalho de Soares de Mello et al. (2004)).

Também deve ser mencionado que, como destacado por Soares de Mello et al. (2004), o modelo de suavização permite eliminar dois dos grandes problemas em DEA: regiões Pareto ineficientes e faces de dimensão não completa. O primeiro devido à existência de uma restrição de monotonicidade, e o segundo pelo facto de a fronteira ser descrita por uma única equação polinomial.

Como mostrado neste trabalho, após alguns desenvolvimentos, as contribuições do modelo proposto por Brandão \& Soares de Mello (2017) trazem oportunidades para tratar inclusive de distorções presentes nos modelos DEA clássicos.

Após todos os estudos e as diversas melhorias propostas, a metodologia de Suavização da Fronteira para modelos BCC tornou-se mais robusta e coerente. No entanto, não é constatada a mesma evolução para os modelos CCR, que ainda apresentam importantes lacunas para serem tratadas em estudos futuros.

Observa-se que o modelo de suavização apresenta cálculos relativamente simples para problemas de pequena dimensão (número de inputs, outputs e DMUs extremoeficientes). Porém, o aumento da dimensão leva a um aumento da quantidade de cálculos algébricos. Em conformidade com as recomendações realizadas pelos autores citados, sugere-se a construção de um software específico para resolução de problemas de Suavização da Fronteira.

\section{REFERÊNCIAS BIBLIOGRÁFICAS}

[1] ALVARENGA, E. F. S. DE; JUNIOR, L. A. M. DE S.; LINS, M. E. Selection of corporate spare parts inventory for Brazilian refineries: a smoothed data envelopment analysis frontier function using calculus of variations. Journal of the Operational Research Society, v. 69, n. 3, p. 392-401, 4 mar. 2018.

[2] ANDERSEN, P.; PETERSEN, N. C. A Procedure for Ranking Efficient Units in Data Envelopment Analysis. Management Science, v. 39, n. 10, p. 1261-1264, 1993.

[3] BANKER, R. D.; CHARNES, A.; COOPER, W. W. Some Models for Estimating Technical and Scale Inefficiencies in Data Envelopment Analysis. Management Science, v. 30, n. 9, p. 1078-1092, 1 set. 1984. 
[4] BRANDÃO, L. C.; SOARES DE MELLO, J. C. C. B. Improvements to smooth data envelopment analysis. RAIRO - Operations Research, v. 51, n. 1, p. 157-171, 1 jan. 2017.

[5] CHARNES, A. et al. Foundations of data envelopment analysis for Pareto-Koopmans efficient empirical production functions. Journal of Econometrics, v. 30, n. 1, p. 91107, 1 out. 1985.

[6] DOYLE, J. R.; GREEN, R. H. Cross-Evaluation In Dea: Improving Discrimination Among Dmus. INFOR: Information Systems and Operational Research, v. 33, n. 3, p. 205-222, 1 ago. 1995.

[7] LINS, M. P. E.; ANGULO MEZA, L. Análise envoltória de dados: perspectivas de integração no ambiente do Apoio à Decisão. [s.l.] Editora da COPPE-UFRJ, 2000.

[8] NACIF, F. B.; SOARES DE MELLO, J. C. C. B.; ANGULO MEZA, L. Choosing weights in optimal solutions for DEA-BCC models by means of a N-dimensional smooth frontier. Pesquisa Operacional, v. 29, n. 3, p. 623-642, dez. 2009.

[9] PEREIRA, E. R. et al. Uso da suavização da fronteira na determinação de pesos únicos em modelos DEA CCR. Production, v. 25, n. 3, p. 585-597, set. 2015.

[10] SOARES DE MELLO, J. C. C. B. et al. Suavização da Fronteira DEA: o Caso BCC Tridimensional. Investigação Operacional, v. 24, n. 1, p. 89-107, jun. 2004.

[11] SOARES DE MELLO, J. C. C. B.; LINS, M. P. E.; GOMES, E. G. Contruction of a smoothed DEA frontier. Pesquisa Operacional, v. 22, n. 2, p. 183-201, dez. 2002.

[12] THANASSOULIS, E. A Comparison of Regression Analysis and Data Envelopment Analysis as Alternative Methods for Performance Assessments. Journal of the Operational Research Society, v. 44, n. 11, p. 1129-1144, 1 nov. 1993. 